\title{
The Cauchy Problem of Couple-Stress Elasticity
}

\author{
O. Makhmudov, I. Niyozov, and N. Tarkhanov
}

This paper is dedicated to our advisors.

\begin{abstract}
We study the Cauchy problem for the oscillation equation of the couple-stress theory of elasticity in a bounded domain in $\mathbb{R}^{3}$. Both the displacement and stress are given on a part $\mathcal{S}$ of the boundary of the domain. This problem is densely solvable while data of compact support in the interior of $\mathcal{S}$ fail to belong to the range of the problem. Hence the problem is ill-posed which makes the standard calculi of Fourier integral operators inapplicable. If $\mathcal{S}$ is real analytic the Cauchy-Kovalevskaya theorem applies to guarantee the existence of a local solution. We invoke the special structure of the oscillation equation to derive explicit conditions of global solvability and an approximation solution.
\end{abstract}

\section{Contents}

1. Introduction

2. Couple-stress elasticity 3

3. Fundamental solution $\quad 5$

4. Somigliana formulas $\quad 7$

5. The Cauchy problem 8

6. Doubly orthogonal systems 10

7. Carleman formula 12

$\begin{array}{ll}\text { References } & 15\end{array}$

\section{Introduction}

The theory of Shapiro-Lopatinskii elliptic boundary value problems on compact smooth manifolds with boundary was well understood by the end of the 1980s.

2000 Mathematics Subject Classification. Primary 74Bxx; Secondary 35 J65.

Key words and phrases. Couple-stress elasticity, oscillation equation, Cauchy problem.

The first author gratefully acknowledges the financial support of the Deutscher Akademischer Austauschdienst.

(C)2000 American Mathematical Society 
While requiring additional investigations, any concrete elliptic problem can be handled within a calculus of pseudodifferential operators which is a slight modification of the Boutet de Monvel algebra.

Beginning with a well-known paper of Kondrat'ev (1967) an actual problem of modern analysis consists in developing diverse pseudodifferential calculi on manifolds with singularities. One of the most advanced theories here is the edge algebra of Schulze [Sch98] who actually adopted many ingredients of the Boutet de Monvel algebra.

In spite of a large number of papers in this direction the main problem of the theory remains still open. It concerns the invertibility of operator-valued symbols which characterizes the Fredholm property of operators in the algebra. This problem may happen to stem from the imperfection of general theory, for real singularities of the physical world have sufficiently many simmetries to reduce the operator-valued symbols.

The Cauchy problem for solutions of elliptic equations is actual since the 1950s when it encountered in geophysics. If the Cauchy data are posed on an open part of the boundary then the Cauchy problem has at most one solution. However, the solution fails to depend continuously on the Cauchy data, unless they are controlled on the whole boundary. In natural setting the Cauchy problem for elliptic equations is ill posed, and the character of instability is similar to that in the problem of analytic continuation.

When compared with Shapiro-Lopatinskii elliptic boundary value problems, the Cauchy problem for elliptic equations bears the characteristic peculiarity of being conditionally stable. The abstract contents of conditional stability are topological vector spaces with two-norm convergence. Classical approaches to the Cauchy problem are discussed and developed in the book [Tar95], to which we refer the reader for the complete bibliography.

We may thus conclude that the Cauchy problem for elliptic equations has never been in the centre of mathematical interests, for it requires non-standard sophisticated tools. The very development of the modern analysis still leads to specifying the Cauchy problem within a calculus of parameter-dependent elliptic boundary value problems, cf. [ST05]. This idea goes back at least as far as that of a boundary layer by Prandtl (1904).

A regular perturbation of the Cauchy problem for elliptic equations suggested in [ST05] gives a mixed boundary value problem of Zaremba type. This brings together two areas of modern analysis in which small parameter methods are of crucial importance, namely, the Cauchy problem for elliptic equations and calculus on manifolds with edges.

The purpose of the present paper is to apply the approach of [Tar95] to study the Cauchy problem for equations of the couple-stress elasticity theory, see for instance [KGBB79]. A great deal of attention is given to questions of the actual construction of solutions in a form allowing one to express them numerically under very general conditions.

A crucial point in constructing explicit approximate solutions is an expansion of the fundamental solution $\Phi(x-y)$ of couple-stress elasticity. The method of [Yar04] seems to extend to derive such an expansion for conic domains. We make use of expansions in eigenfunctions of selfadjoint elliptic operators which are of more general nature. 
For the Cauchy-Riemann operator similar results were recently presented by A. Shlapunov in the Conference on Complex Analysis and Dynamical Systems in Naharia (Israel, January 2-6, 2006).

\section{Couple-stress elasticity}

In the couple-stress theory of elasticity the following approach is adopted, cf. [KGBB79]. The deformed medium is first considered as a set of material particles and the required relations are derived. After that, the medium is idealized, i.e., it is represented as a continuous set, and the techniques of the mathematical analysis are applied.

Let us consider an arbitrary particle with the gravity centre at the point $x$. The particle is assumed to be a rigid body. Its motion is determined by six scalars, e.g., by the displacement of the point $x$ and by the rotation of the particle about its gravity centre.

At the time $t$ the deformed medium will occupy a new position with respect to a fixed system. The point $x$ will move to the position $x^{\prime}$. The displacement vector of $x$ will be determined by $x^{\prime}-x=u(t, x)$. The mobile system will also take a new position with respect to the fixed one. Its rotation angles are denoted by $v_{j}(t, x)$ for $j=1,2,3$. The vector $v(t, x)$ with these coordinates is called the internal rotation of the point $x$ at the time $t$.

If now the medium is assumed to be a continuous one, the motion of each point will be specified not by three scalars, i.e., not by the components of the displacement vector, but by six scalars, i.e., by the components of the displacement and rotation vectors.

The elastic properties of the medium are described by a specific dependence existing between stresses and strains or, more precisely, between the quantities characterizing the stressed and deformed states of the medium. It obeys Hooke's law saying that the deformations of the medium are linear combinations of the stresses applied to it.

In the sequel it will always be assumed that the media under consideration are isotropic and homogeneous with respect to the elastic properties and have the centre of symmetry.

From the mathematical viewpoint an elastic medium may be thought of as a given domain $\mathcal{X}$ of the three-dimensional Euclidean space occupied by this medium in the time interval $\left[t_{0}, T\right]$, and as a given set of constants $\lambda, \mu, \alpha, \rho, \varepsilon, \nu, \beta, \sigma$, satisfying the conditions

$$
\begin{aligned}
& \mu>0, \quad \alpha>0, \quad 3 \lambda+2 \mu>0, \quad \rho>0 \\
& \nu>0, \quad \beta>0, \quad 3 \varepsilon+2 \nu>0, \quad \sigma>0 .
\end{aligned}
$$

Combining the equations of the elastic oscillation state and Hooke's law, cf. [KGBB79, p. 50], we obtain the equation of the elastic oscillation state of the medium, corresponding to the mass force $f$, the mass moment $g$ and the oscillation frequency $\omega$ :

$$
\begin{aligned}
(\mu+\alpha) \Delta u+(\lambda+\mu-\alpha) \nabla \operatorname{div} u+2 \alpha \operatorname{rot} v+\rho \omega^{2} u+\rho f & =0 \\
(\nu+\beta) \Delta v+(\varepsilon+\nu-\beta) \nabla \operatorname{div} v+2 \alpha \operatorname{rot} u-4 \alpha v+\sigma \omega^{2} v+\rho g & =0 .
\end{aligned}
$$


It is convenient to rewrite the basic equations in a matrix form. To this end we introduce a block-matrix

$$
M=\left(\begin{array}{ll}
M^{(1,1)} & M^{(1,2)} \\
M^{(2,1)} & M^{(2,2)}
\end{array}\right)
$$

whose entries are $(3 \times 3)$-matrices of differential operators given by

$$
\begin{aligned}
M_{i, j}^{(1,1)} & =\delta_{i, j}(\mu+\alpha)\left(\Delta+\omega_{1}^{2}\right)+(\lambda+\mu-\alpha) \frac{\partial^{2}}{\partial x_{i} \partial x_{j}} \\
M_{i, j}^{(1,2)} & =M_{i, j}^{(2,1)}=-2 \alpha \sum_{k=1}^{3} \varepsilon_{i, j, k} \frac{\partial}{\partial x_{k}}, \\
M_{i, j}^{(2,2)} & =\delta_{i, j}(\nu+\beta)\left(\Delta+\omega_{2}^{2}\right)+(\varepsilon+\nu-\beta) \frac{\partial^{2}}{\partial x_{i} \partial x_{j}}
\end{aligned}
$$

for $i, j=1,2,3$, where $\omega_{1}^{2}=\frac{\rho \omega^{2}}{\mu+\alpha}$ and $\omega_{2}^{2}=\frac{\sigma \omega^{2}-4 \alpha}{\nu+\beta}$.

Note that the number $\omega_{1}^{2}$ is always non-negative while $\omega_{2}^{2}$ may assume any real value.

By the $\varepsilon$-tensor, also called Levi-Civita's symbol $\varepsilon_{i, j, k}$, is meant $\varepsilon_{i, j, k}=1$ or $\varepsilon_{i, j, k}=-1$ depending on whether $i, j, k$ have an even or odd number of transpositions of the numbers $1,2,3$, and $\varepsilon_{i, j, k}=0$ if at least two of the three indices $i, j, k$ are equal.

Then the equation of the elastic oscillation state of the medium corresponding to the mass force $f$ and the mass moment $g$ becomes

$$
M U+\rho F=0,
$$

the frequency $\omega$ being an arbitrary real number and

$$
U=\left(\begin{array}{l}
u \\
v
\end{array}\right), F=\left(\begin{array}{l}
f \\
g
\end{array}\right) .
$$

We now introduce the stress operator of the couple-stress theory. For this purpose, let $n(x)=\left(n_{1}(x), n_{2}(x), n_{3}(x)\right)$ be an arbitrary unit vector at a point $x$ of the medium. Set

$$
T=\left(\begin{array}{cc}
T^{(1,1)} & T^{(1,2)} \\
T^{(2,1)} & T^{(2,2)}
\end{array}\right),
$$

each entry actually being an $(3 \times 3)$-matrix of differential operators given by

$$
\begin{aligned}
T_{i, j}^{(1,1)} & =\delta_{i, j}(\mu+\alpha) \frac{\partial}{\partial n}+\lambda n_{i} \frac{\partial}{\partial x_{j}}+(\mu-\alpha) n_{j} \frac{\partial}{\partial x_{i}}, \\
T_{i, j}^{(1,2)} & =-2 \alpha \sum_{k=1}^{3} \varepsilon_{i, j, k} n_{k}, \\
T_{i, j}^{(2,1)} & =0, \\
T_{i, j}^{(2,2)} & =\delta_{i, j}(\nu+\beta) \frac{\partial}{\partial n}+\varepsilon n_{i} \frac{\partial}{\partial x_{j}}+(\nu-\beta) n_{j} \frac{\partial}{\partial x_{i}}
\end{aligned}
$$

for $i, j=1,2,3$.

The main purpose of the couple-stress elasticity theory is to determine the elastic oscillation state. The state should continuously depend on the boundary data. The matter is that these data are obtained by measurements and therefore they always differ from their exact values. Hence the concrete state which has been 
found by such approximate data will be of practical importance if it differs from the true state to the same extent as the data differ from their exact values. The problem thus posed is called correct.

In the couple-stress elasticity theory four main problems of oscillation are considered. They consist in finding the elastic oscillation state of the medium if on the boundary we are given the displacements and rotations in the first problem, the force- and couple-stresses in the second problem, the displacements and couplestresses in the third problem, the rotations and force-stresses in the fourth problem, cf. [KGBB79, Ch. IX].

However, non-correctly posed problems are frequently encountered in mathematical physics. As but one example we show the Cauchy problem for solutions of equations (2.2) with data on a proper part of the boundary of $\mathcal{X}$. The interest in such problems has increased in the recent years, cf. [Tar95] et al. They are investigated by using solutions of some correct problems specially constructed for this purpose, cf. [ST05].

\section{Fundamental solution}

The homogeneous equation of steady-state oscillations of the couple-stress theory has the form $M U=0$. By the (two-sided) fundamental solution of convolution type for $M$ is meant any $(6 \times 6)$-matrix $\Phi$ whose entries are distributions in all of $\mathbb{R}^{3}$, such that $M(\Phi * U)=U$ and $\Phi *(M U)=U$ holds for each $C^{\infty}$ function $U$ with compact support and values in $\mathbb{R}^{6}$. A familiar argument shows that this just amounts to saying that

$$
\begin{aligned}
M\left(D_{x}\right) \Phi(x-y) & =\delta(x-y) E_{6}, \\
M^{\prime}\left(D_{y}\right)(\Phi(x-y))^{T} & =\delta(x-y) E_{6}
\end{aligned}
$$

for all $(x, y) \in \mathbb{R}^{3} \times \mathbb{R}^{3}$, where $M^{\prime}$ stands for the transposed differential operator of $M, \Phi^{T}$ for the transposed matrix of $\Phi$, and $\delta$ for the Dirac functional supported at the origin.

Such a fundamental solution can be obtained by the formula $\Phi=M^{C} \varphi$ where $M^{C}$ is the complementary matrix of $M$, i.e., the matrix satisfying the equations $M^{C} M=M M^{C}=(\operatorname{det} M) E_{6}$, and $\varphi$ a fundamental solution of convolution type for the scalar differential operator $\operatorname{det} M$. An elementary, though cumbersome, computation shows that

$$
\operatorname{det} M=c\left(\Delta+k_{1}^{2}\right)\left(\Delta+k_{2}^{2}\right)\left(\Delta+k_{3}^{2}\right)^{2}\left(\Delta+k_{4}^{2}\right)^{2},
$$

where

$$
\begin{aligned}
c & =(\mu+\alpha)^{2}(\lambda+2 \mu)(\nu+\beta)^{2}(\varepsilon+2 \nu) \\
& >0
\end{aligned}
$$

and

$$
k_{1}^{2}=\frac{\rho \omega^{2}}{\lambda+2 \mu}, \quad k_{2}^{2}=\frac{\sigma \omega^{2}-4 \alpha}{\varepsilon+2 \nu},
$$

$k_{3}^{2}$ and $k_{4}^{2}$ satisfy the conditions

$$
\begin{aligned}
k_{3}^{2}+k_{4}^{2} & =\omega_{1}^{2}+\omega_{2}^{2}+\frac{4 \alpha^{2}}{(\mu+\alpha)(\nu+\beta)}, \\
k_{3}^{2} k_{4}^{2} & =\omega_{1}^{2} \omega_{2}^{2} .
\end{aligned}
$$


For an explicit formula for the complementary matrix $M^{C}$ of $M$ we refer the reader to [KGBB79, p. 90]. The formula makes it obvious that $M^{C}(D)$ as well as $M(D)$ is a formally selfadjoint operator.

Since each entry of the matrix $M^{C}$ contains the factor $c\left(\Delta+k_{3}^{2}\right)\left(\Delta+k_{4}^{2}\right)$, it is in fact sufficient to find the mere distribution $c\left(\Delta+k_{3}^{2}\right)\left(\Delta+k_{4}^{2}\right) \varphi$. This latter has the form

$$
e(x)=\sum_{j=1}^{4} C_{j} \frac{-1}{4 \pi} \frac{\exp \left(\imath k_{j}|x|\right)}{|x|}
$$

where

$$
C_{j}=\prod_{\substack{i=1, \ldots, 4 \\ i \neq j}} \frac{1}{\left(k_{i}^{2}-k_{j}^{2}\right)}
$$

cf. [KGBB79, p. 91].

Summarizing we obtain a fundamental solution of convolution type for $M$ on $\mathbb{R}^{3}$ of the form

$$
\Phi(x)=\left(\begin{array}{ll}
\Phi^{(1,1)}(x) & \Phi^{(1,2)}(x) \\
\Phi^{(2,1)}(x) & \Phi^{(2,2)}(x)
\end{array}\right)
$$

whose entries are $(3 \times 3)$-matrices of distributions defined by

$$
\begin{aligned}
\Phi_{i, j}^{(1,1)}(x) & =\sum_{l=1}^{4}\left(\delta_{i, j} a_{l}+b_{l} \frac{\partial^{2}}{\partial x_{i} \partial x_{j}}\right) \frac{-1}{4 \pi} \frac{\exp \left(\imath k_{l}|x|\right)}{|x|} \\
\Phi_{i, j}^{(1,2)}(x) & =\Phi_{i, j}^{(2,1)}(x)=\frac{2 \alpha}{\mu+\alpha} \sum_{l=1}^{4} \sum_{m=1}^{3} \varepsilon_{i, j, m} e_{l} \frac{\partial}{\partial x_{m}} \frac{-1}{4 \pi} \frac{\exp \left(\imath k_{l}|x|\right)}{|x|}, \\
\Phi_{i, j}^{(2,2)}(x) & =\sum_{l=1}^{4}\left(\delta_{i, j} c_{l}+d_{l} \frac{\partial^{2}}{\partial x_{i} \partial x_{j}}\right) \frac{-1}{4 \pi} \frac{\exp \left(\imath k_{l}|x|\right)}{|x|}
\end{aligned}
$$

for $i, j=1,2,3$, cf. [KGBB79, p. 92]. All the constants entering into these equalities are explicitly evaluated by

$$
\begin{array}{ll}
a_{l}=\frac{(-1)^{l}\left(\delta_{3, l}+\delta_{4, l}\right)\left(\omega_{2}^{2}-k_{l}^{2}\right)}{(\mu+\alpha)\left(k_{3}^{2}-k_{4}^{2}\right)}, & b_{l}=-\frac{\delta_{1, l}}{\rho \omega^{2}}+\frac{a_{l}}{k_{l}^{2}}, \\
c_{l}=\frac{(-1)^{l}\left(\delta_{3, l}+\delta_{4, l}\right)\left(\omega_{1}^{2}-k_{l}^{2}\right)}{(\nu+\beta)\left(k_{3}^{2}-k_{4}^{2}\right)}, & d_{l}=-\frac{\delta_{2, l}}{\sigma \omega^{2}-4 \alpha}+\frac{c_{l}}{k_{l}^{2}},
\end{array}
$$

and $e_{l}=\frac{(-1)^{l}\left(\delta_{3, l}+\delta_{4, l}\right)}{(\nu+\beta)\left(k_{3}^{2}-k_{4}^{2}\right)}$, so that the sums $\sum_{l=1}^{4} b_{l}, \sum_{l=1}^{4} d_{l}$ and $\sum_{l=1}^{4} e_{l}$ vanish.

It will be assumed from now on that $k_{3}^{2} \neq k_{4}^{2}$. In addition, we also require that $\sigma \omega^{2}-4 \alpha \neq 0$.

Set $N=M^{C} / c\left(\Delta+k_{3}^{2}\right)\left(\Delta+k_{4}^{2}\right)$. Then $N$ is a formally selfadjoint differential operator of order 6 with constant coefficients in $\mathbb{R}^{3}$ satisfying $N M=M N=p E_{6}$, where

$$
p(D)=\prod_{j=1}^{4}\left(\Delta+k_{j}^{2}\right)
$$

The distribution (3.1) is a fundamental solution of convolution type of $p(D)$ on $\mathbb{R}^{3}$, and $\Phi=N e$.

REMARK 3.1. If $s$ is a solution of $p s=0$ on an open set $O \subset \mathbb{R}^{3}$ then $U=N s$ is a solution of $M U=0$ on $O$. 


\section{Somigliana formulas}

The formulas of Somigliana first proved in the classical elasticity theory, cf. [Som94], extend to the couple-stress elasticity theory in a natural way. In the modern mathematics they are considered in the framework of more general Green formulas, cf. [Tar95, 9.2.1].

Let $\mathcal{X}$ be a Lyapunov domain in $\mathbb{R}^{3}$, i.e., $\mathcal{X}$ is bounded and the unit outward normal vector $n(y)$ exists at each point $y$ of the boundary and satisfies a Hölder condition on $\mathcal{Y}=\partial \mathcal{X}$.

Lemma 4.1. For all $C^{2}$ functions $U, G$ on $\overline{\mathcal{X}}$ with values in $\mathbb{R}^{6}$ the formula is valid

$$
\int_{\mathcal{Y}}\left(\langle G, T U\rangle_{y}-\langle T G, U\rangle_{y}\right) d s=\int_{\mathcal{X}}\left(\langle G, M U\rangle_{y}-\langle M G, U\rangle_{y}\right) d y
$$

where $T=T(y, D)$ is the stress operator related to the vector field $n(y)$ along the boundary.

Proof. This is a very particular case of general Green formula, cf. Theorem 9.2.7 in [Tar95] and elsewhere.

Note that the pair of boundary operators $\left\{E_{6}, T\right\}$ is a Dirichlet system of order 1 on $\mathcal{Y}$.

As usual, by $d s$ is meant the area measure on the hypersurface $\mathcal{Y}$ induced by the Lebesgue measure in $\mathbb{R}^{3}$. Furthermore, $\langle G, F\rangle_{y}:=(G(y))^{T} F(y)$ stands for the pointwise pairing of the values of $G$ and $F$ in $\mathbb{R}^{6}$. We now substitute the rows of the fundamental solution $\Phi(x-\cdot)$ for $G$. To this end, we need the symmetry property of the kernel $\Phi(x-y)$.

Lemma 4.2. The fundamental solution $\Phi(x-y)$ is symmetric in the sense that $(\Phi(x-y))^{T}=\Phi(y-x)$ over $\mathbb{R}^{3} \times \mathbb{R}^{3}$.

Proof. Using the formal selfadjointness of $N$ and the symmetry of $e(x-y)$ we readily get

$$
\begin{aligned}
(\Phi(x-y))^{T} & =\left(N\left(D_{x}\right) e(x-y)\right)^{T} \\
& =\left(N^{*}\left(D_{x}\right) e(x-y)\right)^{T} \\
& =N\left(-D_{x}\right) e(x-y) \\
& =N\left(D_{y}\right) e(x-y) \\
& =N\left(D_{y}\right) e(y-x) \\
& =\Phi(y-x)
\end{aligned}
$$

for all $(x, y) \in \mathbb{R}^{3} \times \mathbb{R}^{3}$, as desired.

Combining Lemmas 4.1 and 4.2 we arrive at a formula which is referred to in [KGBB79, p. 491] as the Somigliana formula.

THEOREM 4.3. Suppose $U$ is a smooth function on the closure of $\mathcal{X}$ with values in $\mathbb{R}^{6}$, such that $M U$ is integrable on $\mathcal{X}$. Then

$$
\int_{\mathcal{Y}}\left((T \Phi(\cdot-x))^{T} U-\Phi(x-\cdot) T U\right) d s+\int_{\mathcal{X}} \Phi(x-\cdot) M U d y= \begin{cases}U(x), & \text { if } x \in \mathcal{X} \\ 0, & \text { if } x \notin \overline{\mathcal{X}}\end{cases}
$$


Proof. We restrict ourselves to formal details only, for the techniques of proving such formulas is well elaborated. We substitute the rows of $\Phi(x-\cdot)$ for $G$ to Lemma 4.1, i.e., take $G=(\Phi(x-\cdot))^{T}$. This yields

$\int_{\mathcal{Y}}\left(\Phi(x-\cdot) T U-(T \Phi(\cdot-x))^{T} U\right) d s=\int_{\mathcal{X}}\left(\Phi(x-\cdot) M U-\left(M^{\prime}(\Phi(x-\cdot))^{T}\right)^{T} U\right) d y$

for all $x$ away from the boundary of $\mathcal{X}$. Since $\Phi(x-y)$ is a left fundamental solution of $M$, it follows that $M^{\prime}(\Phi(x-\cdot))^{T}=\delta(\cdot-x) E_{6}$ holds, which establishes the desired formula.

This formula may be given yet another form which is more suitable for studying the third and the fourth problems of the couple-stress elasticity theory. To this end, we introduce the operators

$$
D=\left(\begin{array}{cc}
E_{3} & 0 \\
0 & -T^{(2,2)}
\end{array}\right), \quad R=\left(\begin{array}{cc}
T^{(1,1)} & T^{(1,2)} \\
0 & E_{3}
\end{array}\right),
$$

where $T^{(I, J)}$ are block-matrices of the stress operator, cf. (2.4).

Corollary 4.4. Let $U$ be a smooth function on the closure of $\mathcal{X}$ with values in $\mathbb{R}^{6}$, such that $M U$ is integrable on $\mathcal{X}$. Then

$\int_{\mathcal{Y}}\left((R \Phi(\cdot-x))^{T} D U-(D \Phi(\cdot-x))^{T} R U\right) d s+\int_{\mathcal{X}} \Phi(x-\cdot) M U d y= \begin{cases}U(x), & x \in \mathcal{X}, \\ 0, & x \notin \mathcal{X}\end{cases}$

However, this formula does not shed any new light on the Cauchy problem for solutions of $M U+\rho F=0$, for the Cauchy data $\{U, T U\}$ and $\{D U, R U\}$ are easily expressed through each other.

\section{The Cauchy problem}

Let $\mathcal{S}$ be an open piece on the boundary $\mathcal{Y}$ of $\mathcal{X}$. The Cauchy problem for solutions of $M U+\rho F=0$ in $\mathcal{X}$ with data on $\mathcal{S}$ consists in the following. Given functions $U_{0}$ and $U_{1}$ on $S$ with values in $\mathbb{R}^{6}$, find a solution $U$ to $M U+\rho F=0$ in $\mathcal{X}$, such that $U=U_{0}$ and $T U=U_{1}$ on $S$. To study this problem we have to choose function spaces for $U_{0}, U_{1}$ and $U$. A rigorous analysis can be found in [Tar95]. It is a little cumbersome, for the behaviour of the solution near the boundary of $S$ requires a careful study. In order to highlight principal difficulties in the Cauchy problem we restrict our attention to the case where $U_{0}$ and $U_{1}$ are integrable functions on $\mathcal{S}$ of class $C^{1}$ and $C^{0}$, respectively. If exists, the solution $U$ should be smooth up to $S$ in $\mathcal{X}$, and we avoid discussion of weak boundary values of $U$ on $S$. We thus consider the problem

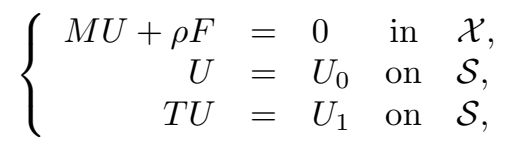

where $F \in L^{q}\left(\mathcal{X}, \mathbb{R}^{6}\right)$ with $q>3$.

It is well known that this problem has at most one solution in any reasonable space of functions on $\mathcal{X}$.

To study (5.1) we introduce an integral completely determined by the Cauchy data $U_{0}$ and $U_{1}$ on $S$ and the right-hand side $-\rho F$ in $\mathcal{X}$, namely

$$
\mathcal{G}\left(U_{0} \oplus U_{1}\right)(x)=\int_{\mathcal{S}}\left((T \Phi(\cdot-x))^{T} U_{0}-\Phi(x-\cdot) U_{1}\right) d s-\int_{\mathcal{X}} \Phi(x-\cdot) \rho F d y
$$


for $x$ away from the closure of $\mathcal{S}$. Since the fundamental solution $\Phi$ is real analytic away from the origin in $\mathbb{R}^{3}$, it follows that $\mathcal{G}\left(U_{0} \oplus U_{1}\right)$ is real analytic in the complement of $\overline{\mathcal{X}}$.

Moreover, the potential (5.2) satisfies $M \mathcal{G}\left(U_{0} \oplus U_{1}\right)=0$ in $\mathbb{R}^{3} \backslash \overline{\mathcal{X}}$. In particular, the components of the vector-valued function $\mathcal{G}\left(U_{0} \oplus U_{1}\right)$ are solutions of the scalar equation $p(D) u=0$ in the complement of $\overline{\mathcal{X}}$.

When $x$ crosses the hypersurface $\mathcal{S}$, both the integral $\mathcal{G}\left(U_{0} \oplus U_{1}\right)$ and its stress $T \mathcal{G}\left(U_{0} \oplus U_{1}\right)$ have jumps. The corresponding jump formulas looks very like the classical Sokhotskii-Plemelj formulas, cf. [KGBB79, p. 492], [Tar95, 10.1.2], and elsewhere.

TheOREM 5.1. In order that there be a solution $U \in C^{1}(\mathcal{X} \cup \mathcal{S})$ of the Cauchy problem (5.1) it is necessary and sufficient that the integral $\mathcal{G}\left(U_{0} \oplus U_{1}\right)$ might be extended from $\mathbb{R}^{3} \backslash \overline{\mathcal{X}}$ through $\mathcal{S}$ to $\mathcal{X}$ as real analytic function.

Proof. Necessity. Suppose there is a solution $U \in C^{1}(\mathcal{X} \cup \mathcal{S})$ of the Cauchy problem (5.1). Define a function $V$ in $\mathbb{R}^{3} \backslash \mathcal{Y}$ by

$$
V=\left\{\begin{array}{lll}
\mathcal{G}\left(U_{0} \oplus U_{1}\right)-U & \text { in } & \mathcal{X} \\
\mathcal{G}\left(U_{0} \oplus U_{1}\right) & \text { in } & \mathbb{R}^{3} \backslash \overline{\mathcal{X}}
\end{array}\right.
$$

Write $V^{ \pm}$for the restriction of $V$ to $\mathcal{X}$ and $\mathbb{R}^{3} \backslash \overline{\mathcal{X}}$, respectively.

We can assume, by shrinking $\mathcal{X}$ if necessary, that $U \in C^{1}\left(\overline{\mathcal{X}}, \mathbb{R}^{6}\right)$. Using Theorem 4.3, we get

$$
V^{+}(x)=-\int_{\mathcal{Y} \backslash \mathcal{S}}\left((T \Phi(\cdot-x))^{T} U-\Phi(\cdot-x) T U\right) d s
$$

for all $x \in \mathcal{X}$. Hence it follows that $V^{+}$extends through $S$ to an analytic function $W$ on all of $\mathbb{R}^{3} \backslash(\mathcal{Y} \backslash \mathcal{S})$ with values in $\mathbb{R}^{6}$.

Once again applying formula (4.1) yields

$$
\begin{aligned}
W(x) & =\int_{\mathcal{S}}\left((T \Phi(\cdot-x))^{T} U-\Phi(\cdot-x) T U\right) d s+\int_{\mathcal{X}} \Phi(x-\cdot) M U d y \\
& =V^{-}(x)
\end{aligned}
$$

for $x \in \mathbb{R}^{3} \backslash \mathcal{X}$. Therefore, $\mathcal{G}\left(U_{0} \oplus U_{1}\right)$ extends from $\mathbb{R}^{3} \backslash \overline{\mathcal{X}}$ through $\mathcal{S}$ to $\mathcal{X}$ as real analytic function, as desired.

Sufficiency. Conversely, let there be a real analytic function $V$ on $\mathbb{R}^{3} \backslash(\mathcal{Y} \backslash \mathcal{S})$ with values in $\mathbb{R}^{6}$, such that $V=\mathcal{G}\left(U_{0} \oplus U_{1}\right)$ away from the closure of $\mathcal{X}$. Then $M V=0$ in $\mathbb{R}^{3} \backslash \overline{\mathcal{X}}$. Since the function $M V$ is real analytic, it actually vanishes in $\mathcal{X}$, too.

Set

$$
U(x)=\mathcal{G}\left(U_{0} \oplus U_{1}\right)(x)-V(x)
$$

for $x \in \mathcal{X}$. From what has already been proved it follows that $U$ is a smooth $\mathbb{R}^{6}$ valued function up to $\mathcal{S}$ in $\mathcal{X}$, satisfying $M U+\rho F=0$. We claim that $U$ is the desired solution of Problem (5.1). To see this, it remains to verify that $U=U_{0}$ and $T U=U_{1}$ on $S$. 
Since $V$ is smooth in $\mathbb{R}^{3} \backslash(\mathcal{Y} \backslash \mathcal{S})$, we readily get by the Sokhotskii-Plemelj formulas

$$
\begin{aligned}
U & =\mathcal{G}\left(U_{0} \oplus U_{1}\right)^{+}-V^{+} \\
& =\mathcal{G}\left(U_{0} \oplus U_{1}\right)^{+}-V^{-} \\
& =\mathcal{G}\left(U_{0} \oplus U_{1}\right)^{+}-\mathcal{G}\left(U_{0} \oplus U_{1}\right)^{-} \\
U_{0} &
\end{aligned}
$$

on $S$. Analogously,

$$
\begin{aligned}
T U & =T \mathcal{G}\left(U_{0} \oplus U_{1}\right)^{+}-T V^{+} \\
& =T \mathcal{G}\left(U_{0} \oplus U_{1}\right)^{+}-T V^{-} \\
& =T \mathcal{G}\left(U_{0} \oplus U_{1}\right)^{+}-T \mathcal{G}\left(U_{0} \oplus U_{1}\right)^{-} \\
U_{1} &
\end{aligned}
$$

on $S$, which is our assertion.

Theorem 5.1 recovers the well-known fact that the Cauchy problem for solutions of elliptic equations with real analytic coefficients is a very particular case of the problem of analytic continuation. This latter is unstable and can only be treated in the framework of calculus of parameter-dependent pseudodifferential operators. The Cauchy problem corresponds to a limit case of such a calculus, cf. [ST05]. The proof of Theorem 5.1 shows that any explicit formula of analytic continuation leads to a formula for solutions of (5.1).

\section{Doubly orthogonal systems}

The "extension problem" for Hilbert spaces of functions has a satisfactory solutions in terms of bases with double orthogonality, cf. [Tar95, Ch. 12]. This idea is due to S. Bergman (1927) who used it, at least in principle, to derive a criterion of analytic continuation.

We apply this method to the Cauchy problem (5.1) in the particular case, where $\mathcal{X}$ is a part of a ball $B=B(0, R)$ with centre at the origin and radius $R>0$, rather than develop the theory in the general case. Let $\mathcal{S}$ be a smooth closed hypersurface in $B$ which does not meet $x=0$ and divides $B$ into two domains. Denote by $\mathcal{X}$ the domain that does not contain the origin. Its boundary $\mathcal{Y}$ consists of $\mathcal{S}$ and a part of the sphere $\partial B$ in $\mathbb{R}^{3}$, cf. Figure 1. The advantage of using domains $\mathcal{X}$ of the

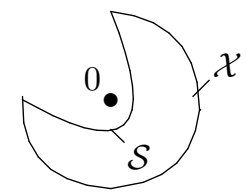

Figure 1. A typical domain.

above form lies in the fact that the problem reduces to analytic continuation from a small ball around 0 to $B$.

As mentioned, the integral $\mathcal{G}\left(U_{0} \oplus U_{1}\right)$ satisfies both $M U=0$ and $p u=0$ away from the closure of $\mathcal{X}$. The latter equation is actually scalar and follows from the former one. Hence we use bases with double orthogonality in Hilbert spaces of 
solutions to $p u=0$ to derive conditions of analytic continuation from $B(0, \varepsilon)$ to $B(0, R)$ for $\mathcal{G}\left(U_{0} \oplus U_{1}\right)$.

The Helmholtz operator $\Delta+k^{2}$ in the space $\mathbb{R}^{3}$ takes in the spherical coordinates the form

$$
\Delta+k^{2}=\frac{1}{r^{2}}\left(\left(r \frac{\partial}{\partial r}\right)^{2}+r \frac{\partial}{\partial r}+k^{2} r^{2}-\Delta_{\mathbb{S}}\right),
$$

where $\Delta_{\mathbb{S}}$ is the Laplace-Beltrami operator on the unit sphere. Recall that $k^{2}$ is an arbitrary real number.

To solve the homogeneous equation $\left(\Delta+k^{2}\right) u=0$ we use the Fourier method of separation of variables. Writing $u(r, \varphi)=g(r, k) h(\varphi)$ we get two separate equations for $g$ and $h$, namely

$$
\begin{aligned}
\left(\left(r \frac{\partial}{\partial r}\right)^{2}+r \frac{\partial}{\partial r}+k^{2} r^{2}\right) g & =c g \\
\Delta_{\mathbb{S}} h & =c h
\end{aligned}
$$

$c$ being an arbitrary constant.

The second equation has non-zero solutions if and only if $c$ is an eigenvalue of $\Delta_{\mathbb{S}}$. These are well known to be $c=i(i+1)$, for $i=0,1, \ldots$, cf. [TS72] and elsewhere. The corresponding eigenfunctions of $\Delta_{\mathbb{S}}$ are spherical harmonics $h_{i}(\varphi)$ of degree $i$, i.e.,

$$
\Delta_{\mathbb{S}} h_{i}=i(i+1) h_{i}
$$

Consider now the following ordinary differential equation with respect to the variable $r>0$

$$
\left(\left(r \frac{\partial}{\partial r}\right)^{2}+r \frac{\partial}{\partial r}+\left(k^{2} r^{2}-i(i+1)\right)\right) g(r, k)=0 .
$$

This is a version of the Bessel equation, and the space of its solutions is twodimensional.

For example, if $k=0$ then $g(r, 0)=a r^{i}+b r^{-i-1}$ with arbitrary constants $a$ and $b$ is a general solution to (6.3). In this situation any function $r^{i} h_{i}(\varphi)$ is a homogeneous harmonic polynomial. In the general case the space of solutions to (6.3) contains a one-dimensional subspace of functions bounded at the point $r=0$, cf. [TS72].

For $i=0,1, \ldots$, fix a non-zero solution $g_{i}(r, k)$ of $(6.3)$ which is bounded at $r=0$. Then

$$
\left(\Delta+k^{2}\right)\left(g_{i}(r, k) h_{i}(\varphi)\right)=0
$$

on all of $\mathbb{R}^{3}$. Indeed, by (6.1), (6.2) and $(6.3)$ we conclude that this equality holds in $\mathbb{R}^{3} \backslash\{0\}$. We now use the fact that $g_{i}(r, k) h_{i}(\varphi)$ is bounded at the origin to see that (6.4) holds.

It is known that there are exactly $J(i)=2 i+1$ linearly independent spherical harmonics of degree $i$. Pick an orthonormal basis

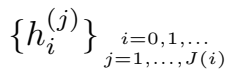

in $L^{2}(\mathbb{S})$.

Lemma 6.1. For every $R>0$, the system

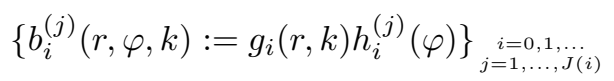

is an orthogonal basis in the subspace of $L^{2}(B(0, R))$ consisting of solutions to the Helmholtz equation $\left(\Delta+k^{2}\right) u=0$. 
Proof. Indeed, as $\left\{h_{i}^{(j)}\right\}$ is an orthonormal basis in the space $L^{2}(\mathbb{S})$ on the unit sphere, the system $(6.5)$ is orthogonal in $L^{2}(B(0, R))$ because

$$
\begin{aligned}
\left(b_{i}^{(j)}, b_{l}^{(m)}\right)_{L^{2}(B(0, R))} & =\left(h_{i}^{(j)}, h_{l}^{(m)}\right)_{L^{2}(\mathbb{S})} \int_{0}^{R} g_{i}(r, k) \overline{g_{l}(r, k)} r^{2} d r \\
& =0
\end{aligned}
$$

for $i \neq l$ or $j \neq m$. Finally, since the system of harmonics $\left\{h_{i}^{(j)}\right\}$ is dense in $C^{\infty}(\mathbb{S})$ we see that system $(6.5)$ is dense in $L^{2}(B(0, R)) \cap \mathcal{S}_{\Delta+k^{2}}(B(0, R))$, which completes the proof.

As a fundamental solution of the operator $\Delta+k^{2}$ in $\mathbb{R}^{3}$ we may choose the standard kernel

$$
\frac{-1}{4 \pi} \frac{\exp (\imath k|x-y|)}{|x-y|}
$$

Given any fixed $y \in \mathbb{R}^{3} \backslash\{0\}$, this kernel is a solution of the Helmholtz equation $\left(\Delta+k^{2}\right) u=0$ in the ball $B(0,|y|)$. Obviously, it is square integrable over $B(0,|y|)$, and we denote by $c_{i}^{(j)}(y, k)$ its Fourier coefficients with respect to the orthogonal system (6.5), i.e.,

$$
c_{i}^{(j)}(y, k)=\left(\frac{-1}{4 \pi} \frac{\exp (\imath k|\cdot-y|)}{|\cdot-y|}, b_{i}^{(j)}(\cdot, k)\right)_{L^{2}(0,|y|)} / \int_{0}^{|y|}\left|g_{i}(r, k)\right|^{2} r^{2} d r .
$$

Lemma 6.2. In the cone $\left\{(x, y) \in \mathbb{R}^{3} \times \mathbb{R}^{3}:|x| /|y|<1\right\}$ a Fourier series expansion is valid

$$
\frac{-1}{4 \pi} \frac{\exp (\imath k|x-y|)}{|x-y|}=\sum_{i=0}^{\infty} \sum_{j=1}^{J(i)} c_{i}^{(j)}(y, k) b_{i}^{(j)}(x, k),
$$

where the series converges uniformly together with all its derivatives on compact subsets of the cone.

Proof. The Fourier series expansion is a direct consequence of Lemma 6.1. The uniform convergence on compact subsets of the cone presents a more delicate problem. It can be handled in a familiar way, for $b_{i}^{(j)}$ are solutions of the Helmholtz equation.

\section{Carleman formula}

We now use expansion (6.6) to study the Cauchy problem of couple-stress elasticity in much the same way as Shlapunov [Shl96] uses an analogous expansion for the fundamental solution of the Laplace equation to investigate the Cauchy problem for the Lamé system.

Namely, substituting (6.6) into the formula for the fundamental matrix $\Phi(x-y)$ of $M$ we obtain

$$
\Phi(x-y)=\sum_{\nu=0}^{\infty} \Phi_{\nu}(x, y)
$$

where the series converges uniformly along with all its derivatives on compact subsets of the cone $\left\{(x, y) \in \mathbb{R}^{3} \times \mathbb{R}^{3}:|x| /|y|<1\right\}$, and $\Phi_{\nu}$ are $(2 \times 2)$-block-matrices 
with entries

$$
\begin{aligned}
\Phi_{\nu ; i, j}^{(1,1)}(x, y) & =\sum_{l=1}^{4}\left(\delta_{i, j} a_{l}+b_{l} \frac{\partial^{2}}{\partial x_{i} \partial x_{j}}\right) \sum_{\mu=1}^{J(\nu)} c_{\nu}^{(\mu)}\left(y, k_{l}\right) b_{\nu}^{(\mu)}\left(x, k_{l}\right), \\
\Phi_{\nu ; i, j}^{(1,2)}(x, y) & =\Phi_{\nu ; i, j}^{(2,1)}(x, y)=\frac{2 \alpha}{\mu+\alpha} \sum_{l=1}^{4} \sum_{m=1}^{3} \varepsilon_{i, j, m} e_{l} \frac{\partial}{\partial x_{m}} \sum_{\mu=1}^{J(\nu)} c_{\nu}^{(\mu)}\left(y, k_{l}\right) b_{\nu}^{(\mu)}\left(x, k_{l}\right), \\
\Phi_{\nu ; i, j}^{(2,2)}(x, y) & =\sum_{l=1}^{4}\left(\delta_{i, j} c_{l}+d_{l} \frac{\partial^{2}}{\partial x_{i} \partial x_{j}}\right) \sum_{\mu=1}^{J(\nu)} c_{\nu}^{(\mu)}\left(y, k_{l}\right) b_{\nu}^{(\mu)}\left(x, k_{l}\right),
\end{aligned}
$$

for $i, j=1,2,3$. We obtain the same formulas if we substitute $-\partial / \partial y_{j}$ for the derivatives $\partial / \partial x_{j}$.

LEMMA 7.1. Every term $\Phi_{\nu}(x, y)$ is a real analytic matrix-valued function on the set $\mathbb{R}^{3} \times\left(\mathbb{R}^{3} \backslash\{0\}\right)$ satisfying

$$
\begin{aligned}
M\left(D_{x}\right) \Phi_{\nu}(x, y) & =0 \\
M^{\prime}\left(D_{y}\right)\left(\Phi_{\nu}(x, y)\right)^{T} & =0 .
\end{aligned}
$$

Proof. These properties are obvious by the very construction of $\Phi_{\nu}(x, y)$. The singularity at $y=0$ is due to

$$
\int_{0}^{|y|}\left|g_{\nu}(r, k)\right|^{2} r^{2} d r
$$

A series expansion like (7.1) with terms $\Phi_{\nu}$ satisfying the transposed equation $M^{\prime}\left(D_{y}\right)\left(\Phi_{\nu}(x, y)\right)^{T}=0$ is already sufficient to derive an explicit formula for solutions of the Cauchy problem (5.1). Set

$$
\mathcal{C}^{(N)}(x, y)=\Phi(x-y)-\sum_{\nu=0}^{N} \Phi_{\nu}(x, y)
$$

for $(x, y) \in \mathbb{R}^{3} \times\left(\mathbb{R}^{3} \backslash\{0\}\right)$. In this way we obtain what is referred to as a Carleman function of the Cauchy problem, cf. [Tar95, 10.4].

Theorem 7.2. Suppose $U$ is a function in $\mathcal{X}$ with values in $\mathbb{R}^{6}$ which is smooth up to the closure of $\mathcal{S}$. Then

$$
U(x)=\lim _{N \rightarrow \infty} \int_{\mathcal{S}}\left(\left(T\left(\mathcal{C}^{(N)}(x, \cdot)\right)^{T}\right)^{T} U-\mathcal{C}^{(N)}(x, \cdot) T U\right) d s+\int_{\mathcal{X}} \mathcal{C}^{(N)}(x, \cdot) M U d y
$$

for all $x \in \mathcal{X}$.

Proof. We can assume, by approximating $\mathcal{X}$ by smaller domains having the only common part $\mathcal{S}$ in the boundary with $\mathcal{X}$, that $U$ is smooth in the closure of $\mathcal{X}$ and $M U$ is integrable over $\mathcal{X}$. Combining Theorem 4.3 with Lemmas 4.1 and 7.1 we easily deduce that

$$
U(x)=\int_{\partial \mathcal{X}}\left(\left(T\left(\mathcal{C}^{(N)}(x, \cdot)\right)^{T}\right)^{T} U-\mathcal{C}^{(N)}(x, \cdot) T U\right) d s+\int_{\mathcal{X}} \mathcal{C}^{(N)}(x, \cdot) M U d y
$$

for each fixed $x \in \mathcal{X}$ and all $N=0,1, \ldots$ Let $N \rightarrow \infty$. Since the series (7.1) converges uniformly along with the first derivatives in $y$ on $\partial B(0, R)$, it follows that the part of the boundary integral over $\partial \mathcal{X} \backslash \mathcal{S}$ tends to zero. This establishes the desired formula. 
Let $U$ be a solution of the Cauchy problem (5.1). A straightforward computation shows that

$$
\begin{aligned}
& \int_{\mathcal{S}}\left(\left(T\left(\mathcal{C}^{(N)}(x, \cdot)\right)^{T}\right)^{T} U-\mathcal{C}^{(N)}(x, \cdot) T U\right) d s+\int_{\mathcal{X}} \mathcal{C}^{(N)}(x, \cdot) M U d y \\
& \quad=\mathcal{G}\left(U_{0} \oplus U_{1}\right)-V_{N}
\end{aligned}
$$

away from the closure of $\mathcal{S}$ in $\mathbb{R}^{3}$, where

$$
V_{N}(x)=\sum_{\nu=0}^{N} \int_{\mathcal{S}}\left(\left(T\left(\Phi_{\nu}(x, \cdot)\right)^{T}\right)^{T} U_{0}-\Phi_{\nu}(x, \cdot) U_{1}\right) d s-\int_{\mathcal{X}} \Phi_{\nu}(x, \cdot) \rho F d y .
$$

Write $\varepsilon>0$ for the distance between $\mathcal{S}$ and the origin. If $x \in B(0, \varepsilon)$ then the left-hand side of (7.2) tends to zero, for the series (7.1) converges uniformly along with the first derivatives in $y$ on $\mathcal{S}$. It follows that the series $\left\{V_{N}\right\}$ converges to $\mathcal{G}\left(U_{0} \oplus U_{1}\right)$ uniformly together with its derivatives on compact subsets of the ball $B(0, \varepsilon)$.

The last observation and Theorem 5.1 fit together to yield certain conditions of solvability for the Cauchy problem.

COROLlaRY 7.3. If the series $\left\{V_{N}\right\}$ converges uniformly on compact subsets of the ball $B(0, R)$ then the Cauchy problem (5.1) is solvable.

Proof. Since the terms of the series $\left\{V_{N}\right\}$ are component-wise solutions of the elliptic equation $p u=0$, it follows by the Stieltijes-Vitali theorem that its sum $V=\lim V_{N}$ satisfies component-wise the same equation in $B(0, R)$. Hence, $V$ is a real analytic function on $B(0, R)$ with values in $\mathbb{R}^{6}$. As $V$ actually coincides with $\mathcal{G}\left(U_{0} \oplus U_{1}\right)$ in the smaller ball $B(0, \varepsilon)$, the solvability of the Cauchy problem follows from Theorem 5.1, as desired.

In many interesting cases the uniform convergence of the series $\left\{V_{N}\right\}$ is not only sufficient but also necessary for the solvability of (5.1), cf. [Shl96, 2.9]. 


\section{References}

[CC98] E. Cosserat and F. Cosserat, Sur le équations de la théorie de l'élasticité, C. R. Acad. Sci., Paris 126 (1898), 1129-1132.

[KGBB79] V. D. Kupradze, T. G. Gegelia, M. O. Basheleishvili, and T. V. Burchuladze, ThreeDimensional Problems of the Mathematical Theory of Elasticity and Thermoelasticity, North-Holland, Amsterdam et al., 1979.

[MN05] O. Makhmudov and I. Niyozov, Regularisation of the Cauchy Problem for the System of Elasticity Theory in $\mathbb{R}^{n}$, Preprint 2005/22, Institut für Mathematik, Universität Potsdam, 2005.

[Sch98] B.-W. Schulze, Boundary Value Problems and Singular Pseudo-Differential Operators, J. Wiley, Chichester, 1998.

[Sh196] A. Shlapunov, Green's Integrals and Their Applications to Elliptic systems, Tesi di perfezionamento, Scuola Normale Superiore, Pisa, 1996, 162 p.

[ST05] A. Shlapunov and N. Tarkhanov, Mixed Problems with a Parameter, Russian J. Math. Phys. 12 (2005), no. 1, 97-119.

[Som94] C. Somigliana, Sue sistemi simmetrici di equazioni a derivate parziali, Ann. Mat. Pura ed Appl. 22 (1894), 143-156.

[Tar95] N. Tarkhanov, The Cauchy Problem for Solutions of Elliptic Equations, Akademie Verlag, Berlin, 1995.

[TS72] A. N. Tikhonov and A. A. Samarskii, Equations of Mathematical Physics, Nauka, Moscow, 1972.

[Yar04] Sh. Yarmukhamedov, A Carleman function and the Cauchy problem for the Laplace equation, Sib. Math. J. 45 (2004), no. 3, 580-595.

(O. Makhmudov) Department of Mechanics and Mathematics, University of SamarKAND, UNIVERSiTy BouleVARd 15, 703004 SAMARKAND, UZBEKISTAN

E-mail address: olimjan@yahoo.com

(I. Niyozov) Department of Mechanics and Mathematics, University of Samarkand, UNIVERSITY BOULEVARD 15, 703004 SAMARKAND, UZBEKISTAN

E-mail address: iqbol@samdu.uz

(N. Tarkhanov) Universität Potsdam, Institut für Mathematik, Postfach 601553 , 14415 Potsdam, Germany

E-mail address: tarkhanov@math.uni-potsdam.de 